\title{
New era of precision plant breeding using genome editing
}

\author{
Jeong-II Kim ${ }^{1}$ Jae-Yean Kim ${ }^{2,3}$ \\ Published online: 28 October 2019 \\ (c) Korean Society for Plant Biotechnology 2019
}

\begin{abstract}
'Genome editing (GE) technology', represented by 'CRISPR/Cas system', became a familiar technology to many biological laboratories, since its beginning in 2011 . GE in the field of agriculture is expected to drive the second green evolution by opening the new era of precision plant breeding. This technology is considered as one of the best novel plant breeding techniques that provide alternative ways to avoid the path of strict regulations of 'genetically modified organisms' or GMOs. Indeed, last February, the first GE product, high oleic soybean oil was successfully commercialized without the regulations applied to GMOs. Thus, it is timely to open a special issue "Plant Genome Editing" that includes review papers and original articles to facilitate communication among scientists who are interested in the plant GE field.

Traditional plant breeding techniques have previously been widely used to improve various crops. However, it is labor-intensive and time-consuming to progress from screening phenotypes and genotypes to the development of commercial varieties. Later, genetic modification (GM) technology has been used to develop value-added crops by the transfer of gene(s) into elite varieties. However, there are unsubstantiated health and environmental safety concerns about the developed GM crops, requiring significant time and costs for the deregulation approval process to be commercialized (Napier et al. 2019). Moreover, the integration of transgenes into the plant genome is random
\end{abstract}

Jeong-Il Kim

kimji@jnu.ac.kr

$\triangle$ Jae-Yean Kim

kimjy@gnu.ac.kr

1 Department of Biotechnology and Kumho Life Science Laboratory, Chonnam National University, Gwangju 61186, Korea

2 Division of Applied Life Science (BK21 Plus Program), Plant Molecular Biology and Biotechnology Research Center, Gyeongsang National University, Jinju 52828, Korea

3 Division of Life Science, Gyeongsang National University, Jinju 52828, Korea and sometimes unstable process in the GM crops. Therefore, more precise breeding techniques are highly required. In these regards, plant GE technology using site-directed nucleases (SDNs), such as zinc-finger nucleases (ZFNs), transcription activator-like effector nucleases (TALENs), and clustered regularly interspaced short palindromic repeats (CRISPR)-associated (Cas) nucleases, might open up new possibilities to accelerate the precise plant breeding to attain desirable traits (Zhang et al. 2019). Especially, the CRISPR/Cas system with CRISPR-associated protein 9 (Cas9) or Cas12a is currently the most popular method for plant GE. In this method, Cas nuclease directed by a single guide RNA (sgRNA) recognizes the target DNA sequence flanked by protospacer adjacent motif (PAM) and generates the site-specific double-strand breaks (DSBs). The resulting DSBs can be repaired by error-prone non-homologous end joining (NHEJ) pathway, often producing nucleotide insertions/deletions (indels) and/or substitutions. Another DSB-repair pathway includes the homology-directed repair (HDR) when homologous donor templates are present at the time of DSB formation.

The new techno-waves by the CRISPR/Cas-based GE not only open a new era in plant precision breeding, but also make major impacts on advancement of basic sciences in plants. The CRISPR-based GE tools are becoming increasingly diversified and sophisticated, including the discovery of various Cas enzymes with unique PAMs and engineering of CRISPR/Cas components for improved GE. The expanding GE toolbox comprises precise Cas9 variants and orthologs, wider genome accessibility by recognizing a simpler PAM, an invention of cytidine or adenine base editors, development of new base mutators, and improved HDRmediated GE techniques. There are also diverse methodologies to deliver the GE tools into the plants, which include Agrobacterium-mediated transformation, polyethylene glycol-mediated protoplast transformation of DNA or ribonucleoprotein (RNP) complexes, viral vector-based delivery, and particle bombardment. Moreover, new approaches such as the haploid inducer-mediated GE (HI-Edit) have recently shown a possibility to accelerate the GE of recalcitrant plant 
varieties. Now, several laboratories are working in the field of plant GE and making much progress in the basic and applied sciences. Therefore, the special issue "Plant Genome Editing" will offer a forum for bringing together investigators with different approaches not only in studying their development and application of molecular scissors for crop development, but also in using of genome editing in plants as basic research tools.

Moreover, how to regulate GE crops and related products is an intensely debated issue at international scientific and political forums. Although the US Department of Agriculture (USDA) concluded that the most GE products would not need the regulation in the last year, many other countries are in the process of drafting the regulatory frameworks for the use of GE plants and related products, mainly depending on the mutations introduced in the plant genome. Basically, GE crops generated using SDNs are divided into four types, SDN-1 generated by NHEJ, SDN-2 or SDN-3 generated by HDR and base substitutions generated by base editors (Jang and Joung 2019). In some countries, it is generally agreed that SDN-1 crops might not need regulation, for example, Australia and Japan (Sato 2019; Thygesen 2019). The case of SDN-2 is rather complicated. In the above two countries, SDN2 GE crops using a recombinant DNA template are regulated as a GMO in principle. However, Japan might regulate SDN-2 crops case-by-case, opening a possibility; if the mutations naturally occur, it would not need the regulation. In contrast to SDN2 or SDN3, base editors do not require a DNA template, thus it might be considered as SDN-1 in Japan. Since many countries including Korea, China and India are preparing the regulation policies for GE-mediated precision breeding products, it will be necessary to monitor the governance of plant GE and related products in the world.

In this special issue, four review articles are included. First, Shelake et al. (2019) highlighted the recent advancements in CRISPR-based mutagenesis tools that enable diverse applications in genetic manipulations, which include gene knockout, gene replacement, targeted base substitutions, and nucleotide diversification of user-defined sites. Second, Han and Kim (2019) reviewed recent advances in developing herbicide-resistant plants by the CRISPR/ Cas9-mediated GE technology. Third, Ahmed et al. (2019) reviewed the current status, challenges, and prospects of GE of different crops in China by highlighting the advanced GE tools and methods. Fourth, Meyer and Heimstaedt (2019) described the governance of GE in agriculture by the national institutions and committees from EU members and provide a useful resource to broaden the debates on the future GE regulation policies.

There are eight original research articles covered in this special issue, focusing on GE of different crops. Park et al. (2019) investigated the effectiveness of delivering the
CRISPR/Cas9 RNP complexes to protoplasts of cruciferous cabbage (Brassica oleracea var. capitata), and successfully obtained transgene-free mutant plants of GIGANTEA, a flowering-time regulator gene, to sustain the prolonged vegetative growth by delaying flowering. Jeong et al. (2019) generated an early flowering and vernalization-independent Chinese cabbage (Brassica rapa spp. pekinensis) through CRISPR/Cas9-mediated knock-out of BraFLC2 and Bra$F L C 3$, and identified the function of multi-copy BraFLC genes. This paper also provides a practical GE protocol for Chinese cabbage. Wang et al. (2019) performed a CRISPR/ Cas9-mediated editing of CsWRKY22 to reduce the susceptibility to Xanthomonas citri subsp. citri in Wanjincheng orange (Citrus sinensis L. Osbeck). The edited Wanjincheng oranges showed enhanced resistance against citrus canker, demonstrating that CRISPR/Cas9-targeted GE is an efficient approach for improving disease resistance in citrus. Jung et al. (2019a, b) showed CRISPR/Cas9-mediated gene mutagenesis in rice. Jung et al. (2019a) performed the CRISPR/Cas9-mediated editing of viviparous- 1 (OsVP1) gene to break seed dormancy in rice and obtained transgenefree lines in the $T_{1}$ generation that exhibited faster germination rate without compromising the other agronomic traits compared with wild-type plants under field conditions. Jung et al. (2019b) performed targeted mutagenesis of $F 3^{\prime} H$, $D F R$ and $L D O X$ genes related to anthocyanin biosynthesis in black rice (Oryza sativa L.). The GE mutant lines lacking T-DNA integration were successfully isolated in the T1 generation and showed the changes in seed color and anthocyanin content. Moreover, in this special issue, Kang et al. (2019) provide general guidelines for $\mathrm{C}$ to $\mathrm{T}$ base editing in plants regarding the base editing window, guide RNA length, and choice of efficient promoters by comparing two baseediting systems, APOBEC1-nCas9 and nCas9-PmCDA1, in the protoplasts of Glycine max, Brassica napus, and Nicotiana tabacum. Interestingly, the efficiency of sgRNAs in the protoplast system did not correspond to the actual editing efficiency in agroinfiltration-based tissue culture system. Following the guidelines, the herbicide-resistant $N$. tabacum lines were generated by a specific $\mathrm{C}$ to $\mathrm{T}$ conversion in the acetolactate synthase gene. Yun et al. (2019) introduced a zero-background CRISPR binary vector platform using a $c c d B$-mediated negative selection for construction of sgRNA libraries in plant functional genomics. The authors demonstrated the reliability of this zero-background CRISPR binary platform in a high-throughput pooled cloning of multiple sgRNAs. Finally, Kang (2019) provides the web-application for mining the open reading frame (ORF) of genes from the sugarcane genome. The researchers who want to design guide RNA for sugarcane GE can find the ORF of genes easily with a query gene ID and catalog candidate gene list by query sentences (http://pgl.gnu.ac.kr/sugar cane_orf_finder/). 
In the end, we hope that this special issue on the "Plant Genome Editing" could provide current overviews on the plant GE research and attract further interests of plant scientists in the field.

Acknowledgements We sincerely wish to thank all the contributors and the people in the editorial office of the journal for their help, which made it possible to publish this special issue. We also thank members of the Society of Plant Breeding Innovation (SPBI) for their helpful comments and valuable discussion for the success of this special issue. We thank Dr. Shelake RM for his critical reading and comments. This work was supported by the Next-Generation BioGreen 21 Program from Rural Development Administration, Republic of Korea (TAGC grant no. PJ01325301 and SSAC Grant No. PJ01322601) and partly by the National Research Foundation of Korea (NRF Grant Nos. 2017R1A2B4010349 and 2017R1A4A1015515).

\section{References}

Ahmed S, Zhang Y, Abdullah M, Ma Q, Wang H, Zhang P (2019) Current status, challenges, and future prospects of plant genome editing in China. Plant Biotechnol Rep. https://doi.org/10.1007/ s11816-019-00577-6

Han YJ, Kim JI (2019) Application of CRISPR/Cas9-mediated gene editing for the development of herbicide-resistant plants. Plant Biotechnol Rep. https://doi.org/10.1007/s11816-019-00575-8

Jang G, Joung YH (2019) CRISPR/Cas-mediated genome editing for crop improvement: current applications and future prospects. Plant Biotechnol Rep 13:1-10

Jeong SY, Ahn H, Ryu J, Oh Y, Sivanandhan G, Won K-H, Park YD, Kim J-S, Kim H, Lim YP, Kim SG (2019) Generation of early flowering Chinese cabbage (Brassica rapa ssp. pekinensis) through CRISPR/Cas9-mediated genome editing. Plant Biotechnol Rep. https://doi.org/10.1007/s11816-019-00566-9

Jung YJ, Lee HJ, Bae S, Kim JH, Kim DH, Kim HK, Nam KH, Nogoy FM, Cho YG, Kang KK (2019a) Acquisition of seed dormancy breaking in rice (Oryza sativa L.) via CRISPR/Cas9-targeted mutagenesis of $O s V P 1$ gene. Plant Biotechnol Rep. https://doi. org/10.1007/s11816-019-00580-x

Jung YJ, Lee HJ, Kim JH, Kim DH, Kim HK, Cho Y-G, Bae SS, Kang KK (2019b) CRISPR-Cas9 targeted mutagenesis of $F 3^{\prime} H, D F R$ and $L D O X$ genes related to anthocyanin biosynthesis in black rice (Oryza sativa L.). Plant Biotechnol Rep (in press)
Kang YJ (2019) Sugarcane ORF finder: the web-application for mining genes from sugar cane genome. Plant Biotechnol Rep. https://doi. org/10.1007/s11816-019-00574-9

Kang B-C, Woo JW, Kim S-T, Bae S-J, Choi M, Kim J-S, Kim S-G (2019) Guidelines for $C$ to $T$ base editing in plants: base editing window, guide RNA length, and efficient promoter. Plant Biotechnol Rep. https://doi.org/10.1007/s11816-019-00572-x

Meyer M, Heimstaedt C (2019) The divergent governance of gene editing in agriculture: a comparison of institutional reports from seven EU member states. Plant Biotechnol Rep. https://doi.org/10.1007/ s11816-019-00578-5

Napier JA, Haslam RP, Tsalavouta M, Sayanova O (2019) The challenges of delivering genetically modified crops with nutritional enhancement traits. Nat Plants 5:563-567

Park SC, Park S, Jeong YJ, Lee SB, Pyun JW, Kim S, Kim TH, Kim SW, Jeong JC, Kim CY (2019) DNA-free mutagenesis of GIGANTEA in Brassica oleracea var. capitata using CRISPR/ Cas9 ribonucleoprotein complexes. Plant Biotechnol Rep. https ://doi.org/10.1007/s11816-019-00585-6

Sato S (2019) Japanese Health Ministry finalizes genome edited food policy. USDA Foreign Agricultural Service GAIN Report JA9050

Shelake RM, Pramanik D, Kim JY (2019) Evolution of plant mutagenesis tools: a shifting paradigm from random to targeted genome editing. Plant Biotechnol Rep. https://doi.org/10.1007/s1181 6-019-00562-Z

Thygesen P (2019) Clarifying the regulation of genome editing in Australia: situation for genetically modified organisms. Transgenic Res 28:151-159

Wang L, Chen S, Peng A, Xie Z, He Y, Zou X (2019) CRISPR/Cas9mediated editing of CsWRKY22 reduces susceptibility to Xanthomonas citri subsp. citri in Wanjincheng orange (Citrus sinensis (L.) Osbeck). Plant Biotechnol Rep. https://doi.org/10.1007/s1181 6-019-00556-x

Yun J-Y, Kim S-T, Kim S-G, Kim J-S (2019) A zero-background CRISPR binary vector system for construction of sgRNA libraries in plant functional genomics applications. Plant Biotechnol Rep. https://doi.org/10.1007/s11816-019-00567-8

Zhang Y, Malzahn AA, Sretenovic S, Qi Y (2019) The emerging and uncultivated potential of CRISPR technology in plant science. Nat Plants 5:778-794

Publisher's Note Springer Nature remains neutral with regard to jurisdictional claims in published maps and institutional affiliations. 